\title{
Worked-out examples of the adequacy of Bayesian optional stopping
}

\author{
Jorge N. Tendeiro - Henk A. L. Kiers . \\ Don van Ravenzwaaij
}

This is a post-peer-review, pre-copyedit version of an article published in Psychonomic Bulletin \& Review. The final authenticated version will be/is available online at http://dx.doi.org/10.3758/s13423-021-01962-5

Received: date / Accepted: date

\begin{abstract}
The practice of sequentially testing a null hypothesis as data are collected until the null hypothesis is rejected is known as optional stopping. It is well-known that optional stopping is problematic in the context of $p$-value based null hypothesis significance testing: The false positive rates quickly overcome the single test's significance level. However, the state of affairs under null hypothesis Bayesian testing, where $p$-values are replaced by Bayes factors, has perhaps surprisingly been much less consensual. Rouder (2014) used simulations to defend the use of optional stopping under null hypothesis Bayesian testing. The idea behind these simulations is closely related to the idea of sampling from prior predictive distributions. Deng et al. (2016) and Hendriksen et al. (2020) have provided mathematical evidence to the effect that optional stopping under null hypothesis Bayesian testing does hold under some conditions. These papers are, however, exceedingly technical for most researchers in the applied social sciences. In this paper we provide some mathematical derivations concerning Rouder's approximate simulation results for the two Bayesian hypothesis tests that he considered. The key idea is to consider the probability distribution of the Bayes factor, which is regarded as being a random variable across repeated sampling. This paper therefore offers an intuitive perspective to the literature and we believe it is a valid contribution towards understanding the practice of optional stopping in the context of Bayesian hypothesis testing.
\end{abstract}

Keywords Bayes factor $\cdot$ null hypothesis Bayesian testing $\cdot$ null hypothesis significance testing $\cdot$ sequential testing $\cdot p$-value

\footnotetext{
Jorge N. Tendeiro ${ }^{1,2}$, Henk A. L. Kiers ${ }^{2}$, Don van Ravenzwaaij ${ }^{2}$

${ }^{1}$ Office of Research and Academia-Government-Community Collaboration, Education and Research Center for Artificial Intelligence and Data Innovation, Hiroshima University, Japan. ${ }^{2}$ University of Groningen, The Netherlands.

E-mail: tendeiro@hiroshima-u.ac.jp
} 


\section{Introduction}

Psychological science is living exciting and quickly changing days. From the midst of the crisis of confidence that afflicts our field, it has become clear that part of the solution is to reform null hypothesis significance testing (NHST). There are many literature sources stating the reasons supporting this decision. Here we refer to the recent special issue published in The American Statistician (Wasserstein et al. 2019), consisting of a set of 43 (!) articles that elaborate in detail on these issues.

One may question whether NHST is to be blamed for all the problems in our field. Arguably, some of the problems may be ascribed to practitioners misusing NHST. Questionable research practices (QRPs; John et al. 2012) such as post hoc removing of observations, performing multiple tests but reporting only the very few that 'worked out', and merging conditions are unfortunately all too common (Simmons et al. 2011). In this paper we focus on another such QRP: Collecting more data while continuously testing for significance until rejection of the null hypothesis is possible. One aspect that QRPs make salient is that having the analysis depend on observing the outcome variables of interest (e.g., under optional stopping, or sequential testing, procedures) may have deleterious effects on the quality of the published research.

Proposed solutions to mitigate some of the problems alluded to above include the preregistration of experiments (Nosek et al. 2018), the use of registered reports (Nosek and Lakens 2014), and the adoption of alternative statistical approaches to NHST. Here we explicitly consider one alternative to NHST and $p$-values that has gained increased attention in the literature in recent years, namely, that of null hypothesis Bayesian testing (NHBT) and the Bayes factor (Jeffreys 1961; Kass and Raftery 1995; Tendeiro and Kiers 2019; van de Schoot et al. 2017). Given two models $\mathcal{M}_{0}$ and $\mathcal{M}_{1}$, the Bayes factor $B F_{10}$ is the multiplicative term that updates the prior odds into posterior odds:

$$
\underbrace{\frac{p\left(\mathcal{M}_{1}\right)}{p\left(\mathcal{M}_{0}\right)}}_{\text {prior odds }} \times \underbrace{\frac{p\left(D \mid \mathcal{M}_{1}\right)}{p\left(D \mid \mathcal{M}_{0}\right)}}_{B F_{10}}=\underbrace{\frac{p\left(\mathcal{M}_{1} \mid D\right)}{p\left(\mathcal{M}_{0} \mid D\right)}}_{\text {posterior odds }} .
$$

In other words, the Bayes factor quantifies the update in our relative belief about the two entertained models in light of the observed data $D$. Alternatively, the Bayes factor can be interpreted as the relative predictive value of each model. If, for instance, the observed data are better predicted under $\mathcal{M}_{1}$ then $p\left(D \mid \mathcal{M}_{1}\right)>p\left(D \mid \mathcal{M}_{0}\right)$ and as a consequence $B F_{10}>1$. If $\mathcal{M}_{0}$ represents the null model and $\mathcal{M}_{1}$ an alternative model, the Bayes factor can be used to quantify evidence for $\mathcal{M}_{1}$ compared to $\mathcal{M}_{0}$; if a prior probability ratio is given, a posterior probability ratio can be computed. For a more thorough discussion of Bayes factors, including their merits and possible issues, see Tendeiro and Kiers (2019). 


\subsection{Goal of this paper}

In this paper we consider the problem of optional stopping by means of NHBT. It is well-known that optional stopping is a real problem under $p$-value based NHST (Armitage et al. 1969); we will quickly illustrate the problem with a simple and well-known example. Interestingly, the optional stopping problem (or lack thereof) under the Bayesian framework has been far less consensual. In a provokingly titled paper ('Optional stopping: No problem for Bayesians'), Rouder (2014) suggested that optional stopping is allowed under the Bayesian paradigm (he is not alone; see Edwards et al. 1963; Kadane et al. 1996). Rouder's main premise is that the Bayesian optional stopping procedure leads to a well calibrated decision rule in a particular sense: Given equal prior model probabilities, if for example $B F_{10}=10$ then it must happen that data randomly generated under $\mathcal{M}_{1}$ are 10 times more likely to produce $B F_{10}=10$ than data randomly generated under $\mathcal{M}_{0}$. It is in this particular sense that Rouder (2014) claims that the Bayes factor is able to handle optional stopping (de Heide and Grünwald 2017). Before proceeding, we note that other perspectives on the Bayesian optional stopping problem do exist. At the base of these alternatives is the multiplicity of ways of operationalizing the idea of being "able to handle optional stopping" (de Heide and Grünwald 2017). And in fact, others have argued that the Bayesian optional stopping procedure can be problematic in some sense (e.g., Sanborn et al. 2014; Sanborn and Hills 2014; Yu et al. 2014; de Heide and Grünwald 2017). For the current paper, we will focus exclusively on Rouder's perspective on the optional stopping problem (for those interested, see Rouder and Haaf 2019 for a recent account of Rouder's position on the aforementioned problems).

Recently, Deng et al. (2016) and especially Hendriksen et al. (2020) have offered mathematical proofs showing that the Bayesian optional stopping procedure is well calibrated in the sense of Rouder (2014). These papers further extended the realm of situations under which Bayesian optional stopping is expected to work (including in cases involving improper priors and nuisance parameters). Unfortunately, both these papers are mathematically too technical for the common readership in the applied social sciences.

Rouder (2014) defended his assertions by means of simulated data. For fixed Bayes factors (called 'nominal' Bayes factors), many datasets were generated under either model and the ratio of cases under each model leading to (approximately) that Bayes factor was computed (this is the 'observed' Bayes factor). Our current contribution consists of offering worked-out examples that complement Rouder's conjectures (which are proved in general terms by Hendriksen et al. 2020). Whenever possible, closed-form mathematical derivations of distributions of random variables which complement Rouder's simulated distributions are provided. To the best of our knowledge, our contribution is unique in its modus operandi. Thus, we expect that our approach can offer help to further understand the optional stopping problem under the Bayesian framework, in the sense advocated by Rouder (2014). 
The remainder of the paper is organized as follows. In the next section we introduce the optional stopping procedure in detail, both under the frequentist and the Bayesian paradigms. We motivate Rouder's reasoning by illustrating the connection between his idea and the definition of the Bayes factor as a ratio of two prior predictive distributions (e.g., Etz et al. 2018). Next, we show our results for the two hypotheses tests considered in Rouder (2014). Each section includes theoretical derivations of distributions of the Bayes factor. This is possible by looking at the Bayes factor as a function of randomly drawn data. Thus, effectively, what we offer is sampling distributions for the Bayes factor under either model under comparison. This approach complements that of Rouder, which is exclusively based on simulation. We compare our theoretically derived distributions with approximated distributions found by simulation, thus making clear that our precise results actually strengthen the simulation-based results by Rouder. We finish the paper with a discussion of the main theoretical findings in our derivations and some of the limitations of our approach. Most of the mathematical derivations are included in the supplemental material, to ease the reading of the paper. Furthermore, we provide all the $\mathrm{R}$ code that implements our results and produces all figures in OSF (https://osf.io/5z92h/).

\section{Optional stopping}

\subsection{Under the frequentist paradigm}

In this setting, the optional stopping (or sequential testing) procedure amounts to looking regularly at the statistical significance as data come in, and stopping once the $p$-value falls below the critical threshold $\alpha$ (typically .05 or .01). The problem with this approach is that it leads to proportions of false positive results above the nominal Type I error rate $\alpha$ (typically .05). This is a well known result (e.g., Armitage et al. 1969; Jennison and Turnbull 1990). We used simulation to illustrate the situation. The following procedure was replicated 1,000 times: Randomly draw $n=2$ observations from $\mathcal{N}(0,1)$ and compute the $p$-value of a two-tailed one-sample $t$-test $\left(\mathcal{M}_{0}: \mu=0\right.$ versus $\left.\mathcal{M}_{1}: \mu \neq 0\right)$; stop if $p$ is below $\alpha=.05$, otherwise collect one more observation and recompute $p$; proceed until either $p<\alpha$ or $n$ is 1,000,000. Figure 1 shows the cumulative proportion of replications that terminated due to statistical significance (i.e., a false positive) as a function of the sample size. As can be seen, the proportion of false positives increases ${ }^{1}$ with $n$, and even at low sample sizes the error rates are already deemed unacceptably large (e.g., .36 at $n=50)$. Therefore,

\footnotetext{
1 As a matter of side-interest, we observe that the increase of the false positive rate decelerates as $n$ increases (see Armitage et al. 1969, p. 243), and is approximately loglinear. Thus, the false positive rate is still about $80 \%$ even after (potentially) sampling up to one million observations. The common saying 'sampling to reach a foregone conclusion' (Anscombe 1954) that is often used to criticize the frequentist optional stopping procedure might therefore not accurately describe the situation.
} 


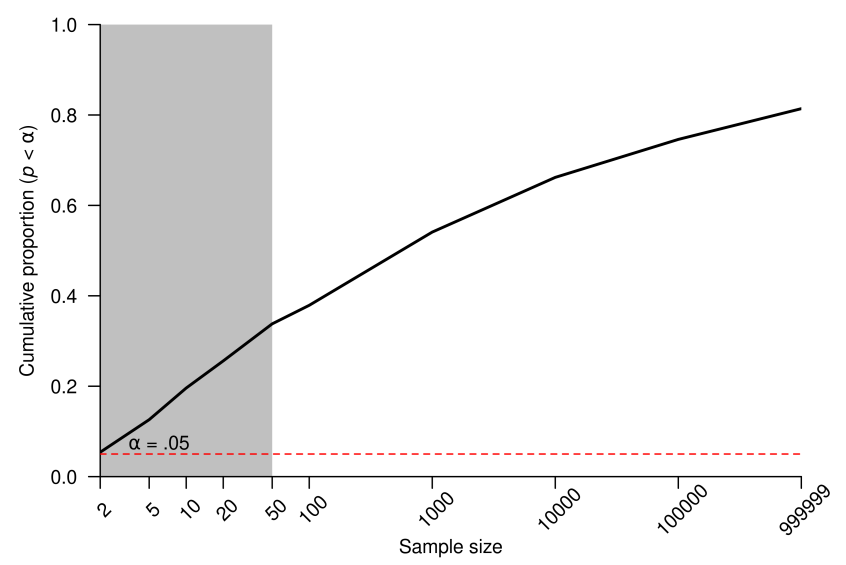

Fig. 1 Proportion of false positives as a function of sample size under the frequentist optional stopping procedure, for a one-sample $t$-test. The $x$-axis is in log-scale. The grayed area shows high agreement with results previously shown by Jennison and Turnbull (1990, Table 1).

optional stopping under the frequentist paradigm leads to serious biases in favor of rejecting the null hypothesis.

Although there are proposals under the frequentist framework to try to remedy the situation (e.g., Armitage 1960; Botella et al. 2006; Fitts 2010; Frick 1998; Jennison and Turnbull 1999; Lakens 2014; Pocock 1983; Wald 1945), these are largely ignored in psychology (Lakens 2014). If such corrections are not used, researchers must adhere to designs based on predefined (i.e., fixed) sample sizes and sample until completion. Unfortunately, there is ample empirical evidence supporting the hypothesis that data collection decisions made by researchers are often dependent on the magnitude of the $p$-value (John et al. 2012; Yu et al. 2014). For example, Yu et al. (2014) found evidence indicating that researchers are willing to prematurely terminate data collection upon observing either a low or a large $p$-value. The problem is severe, and one may rightfully question whether similar limitations hold with Bayesian statistics.

\subsection{Under the Bayesian paradigm}

An optional stopping procedure similar to that under the frequentist paradigm is now considered. Unlike the frequentist procedure, now the $p$-value is replaced by the Bayes factor $B F_{10}$. After observing the $n$-th datum and computing $B F_{10}$, one decides to stop data collection and retain $\mathcal{M}_{1}$ if $B F_{10}$ is larger than a threshold $B F_{U}$ (thus, data evidence is deemed sufficiently compelling for $\mathcal{M}_{1}$ ). Alternatively, one decides to stop data collection and retain $\mathcal{M}_{0}$ if $B F_{10}$ is smaller than a threshold $B F_{L}$ (data evidence is deemed sufficiently compelling for $\left.\mathcal{M}_{0}\right)$. Finally, data collection should proceed in case $B F_{L}<B F_{10}<B F_{U}$. 
The thresholds $B F_{U}$ and $B F_{L}$ should be chosen before data collection. In our analyses we use the same threshold values as Rouder (2014): $B F_{U}=10$ and $B F_{L}=\frac{1}{10}$ (see also Schönbrodt et al. 2017). These values can be considered as strong evidence, according to Jeffreys $(1961)^{2}$. Thus, similar amounts of evidence are required to terminate data collection in favor of either model when Bayesian optional stopping is employed. We notice that the Bayesian optional stopping procedure differs from the frequentist counterpart in one crucial aspect: The Bayesian procedure can stop due to sufficiently strong evidence in favor of $\mathcal{M}_{0}$. This is not possible by means of $p$-value based NHST, where evidence can only be gathered against $\mathcal{M}_{0}$. Thus, the Bayesian optional stopping procedure has this one clear advantage over the frequentist method.

The Bayesian optional stopping procedure outlined above has been recurrently proposed in the literature (as early as Lindley 1957; see also Kass and Raftery 1995; Edwards et al. 1963). Several studies implemented and further extended this method (e.g., Matzke et al. 2015; Schönbrodt et al. 2017; Schönbrodt and Wagenmakers 2018; Wagenmakers et al. 2012, 2015). There is general agreement that, under the Bayesian framework, the stopping rule is unrelated to the interpretation of the results (Rouder 2014; Sanborn and Hills 2014 ); this is a consequence of the likelihood principle (Berger and Wolpert 1988). However, some have questioned frequentist properties related to the procedure, that is, how the procedure works in the long run (Sanborn et al. 2014; Yu et al. 2014). Therefore, the issue of whether the procedure 'works as advertised' has been a matter of contention.

In an attempt to clarify matters, Rouder (2014) presented an argument according to which the Bayes factor is said to be well calibrated under Bayesian optional stopping. To understand the reasoning behind Rouder's simulation study, we make use of the concept of a prior predictive distribution (see e.g., Etz et al. 2018 for a more elaborate exposition). Assume entertaining two competing models in order to infer about the mean $\mu$ of a normally distributed population with known variance $\sigma^{2}: \mathcal{M}_{0}: \mu=0$ versus $\mathcal{M}_{1}: \mu \sim \mathcal{N}\left(0, \sigma_{1}^{2}\right)$ with $\sigma_{1}$ known (the corresponding Bayes factor is worked at length in this paper). We can generate values of $\mu$ from both of these models. ${ }^{3}$ Subsequently, we can generate sample data based on these $\mu$ parameters. The distributions of the resulting sample means (i.e., the prior predictive distributions) for 100, 000 simulations, each simulation consisting on a sample of size 10, are shown in panel A of Figure 2 (sample means larger than .25 in absolute value are not shown to ease the visualization).

The Bayes factor $B F_{10}$ quantifies the relative probability of the observed data under $\mathcal{M}_{1}$ versus $\mathcal{M}_{0}$. In other words, the Bayes factor equals the ratio of the two prior predictive distributions (for an arbitrary sample mean $\bar{X}$ ). Thus, we can compare the relative heights of the two distributions for different points along the $x$-axis to get the corresponding Bayes factors; see panel C.

\footnotetext{
2 We do caution researchers to take qualitative labels such as 'strong' evidence with a grain of salt. Such labels serve no purpose other than benchmarking amounts of evidence.

3 Note that for $\mathcal{M}_{0}$, all generated $\mu$ parameters will be zero.
} 

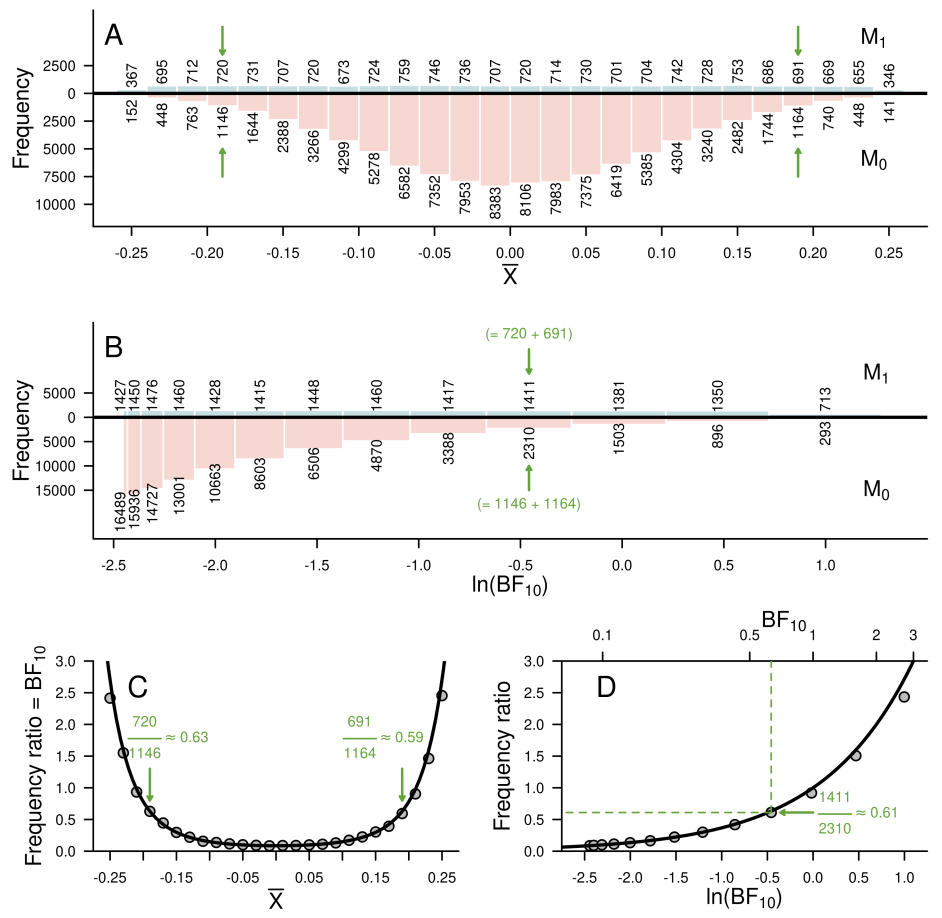

Fig. 2 Prior predictive distributions (panels A and B) and the corresponding ratios (panels $\mathrm{C}$ and D), for normally distributed data $\left(X_{i} \sim \mathcal{N}\left(\mu, \sigma^{2}\right)\right.$ for $i=1, \ldots, n$ and known variance $\left.\sigma^{2}\right)$, when comparing $\mathcal{M}_{0}: \mu=0$ versus $\mathcal{M}_{1}: \mu \sim \mathcal{N}\left(0, \sigma_{1}^{2}\right)$, with $\sigma_{1}$ known. In panels $\mathrm{A}$ and $\mathrm{B}$, the prior predictive distributions for either model are plotted back-to-back for easier visual comparison. The (logarithm of) the Bayes factor is a mathematical function of the sample mean $\bar{X}$, thus we can think of it as being a random variable. The prior predictive distributions for $\bar{X}$ (panel A) and for $\ln \left(B F_{10}\right.$ ) (panel B) under each model are closely related, as indicated by the absolute frequencies. Therefore, the ratios of both pairs of prior predictive distributions give the Bayes factor (panels C and D). See text for details.

The two-sided alternative model $\mathcal{M}_{1}$ is symmetric in the sense that its within-model prior is symmetric around 0. The green arrows in panel A indicate the observed frequencies under either model of sample means of (about) .19 in magnitude. As shown in panel $\mathrm{C}$, the associated Bayes factor is approximately the same $(.63$ at $\bar{X}=-.19$ and .59 at $\bar{X}=.19$; the exact value (solid line) is $\left.B F_{10}=.63\right)$.

Observe that the Bayes factor is a mathematical function of the sample mean (for the running example, see Equation 13). Panel B in Figure 2 displays the frequencies of the (logarithm of) $B F_{10}$. Each bin in panel $\mathrm{B}$ (with a particular $\ln \left(B F_{10}\right)$ value) is matched by the pair of bins in panel $\mathrm{A}$ with sample means corresponding to that $B F_{10}$ value via Equation 13 . For example, $\bar{X}= \pm .19$ corresponds to $\ln \left(B F_{10}\right)=-.46$; the frequency associated to $\ln \left(B F_{10}\right)=-.46$ is the sum of the frequencies associated to $\bar{X}= \pm .19$, under 
each model (see the green arrows in panel B). Their ratio should therefore also equal the Bayes factor; that is displayed in panel D.

More generally, let's consider prior model odds of 1-to-1, thus assume that both models are equally likely a priori. In this case, the Bayes factor is equal to the posterior odds (by Equation 1):

$$
\underbrace{\frac{p\left(D \mid \mathcal{M}_{1}\right)}{p\left(D \mid \mathcal{M}_{0}\right)}}_{B F_{10}}=\underbrace{\frac{p\left(\mathcal{M}_{1} \mid D\right)}{p\left(\mathcal{M}_{0} \mid D\right)}}_{\text {posterior odds }} .
$$

The distribution of the left-hand side of Equation 2 under either $\mathcal{M}_{0}$ or $\mathcal{M}_{1}$ for a given sample size can be approximated by simulation (as illustrated by panel B in Figure 2). But the Bayes factor is equal to the posterior odds by Equation 2. Thus, for each generated data set (under either model) we know that $\mathcal{M}_{1}$ is more likely than $\mathcal{M}_{0}$ by a factor corresponding to the resulting $B F_{10}$ for that data set. Rouder's reasoning is the following (Assertion 1): The previous interpretation must hold even when one does not know under which model the data were generated. In other words, given a data set generated under either $\mathcal{M}_{0}$ or $\mathcal{M}_{1}$ (unknown to us), the Bayes factor can still be interpreted as the corresponding posterior odds. It is in this sense that Rouder claims that the Bayes factor (i.e., the posterior odds) is well calibrated (de Heide and Grünwald 2017 ). Rouder used simulations to make his point. For any given $B F_{10}$ value, he tallied the number of simulations that resulted in approximately ${ }^{4}$ that Bayes factor, under either model (i.e., panel B in Figure 2). The ratio of obtained Bayes factors under both models must then equal the Bayes factor itself under Rouder's assertion (i.e., panel D in Figure 2). For instance, the number of replicate experiments with a fixed sample size that produce $B F_{10}=10$ under $\mathcal{M}_{1}$ should be approximately ten times larger than the number of replicate experiments that produce $B F_{10}=10$ under $\mathcal{M}_{0}$.

Rouder then further suggested the following (Assertion 2): The above property also holds under optional stopping. Thus, using the Bayesian optional stopping procedure in each replication should preserve the property above of the Bayes factor. This goes beyond Assertion 1 in that it lets go of the fixed sample size. Instead, data gets continuously sampled until the $B F_{10}$ hits one of two thresholds (for instance, $B F_{10}=10$ or $B F_{10}=1 / 10$ ). Again, the number of replicate experiments under optional stopping that produce $B F_{10}=10 \mathrm{un}$ der $\mathcal{M}_{1}$ should be approximately ten times larger than the number of replicate experiments that produce $B F_{10}=10$ under $\mathcal{M}_{0}$.

\subsection{Our rationale}

Rouder (2014) provided evidence for his statements by means of simulations and approximations. We now offer a mathematical view on this problem and

\footnotetext{
4 Because the Bayes factor is a continuous measure of evidence, a small interval around $B F_{10}$ has to be considered for this approximation to work.
} 
derive probability distributions instead of approximate histograms of frequencies. We considered the same hypothesis testing procedures as Rouder. These are based on testing hypotheses about the mean of a normal distribution $\mathcal{N}\left(\mu, \sigma^{2}\right)$, for which the variance is assumed known. The first test is given by $\mathcal{M}_{0}: \mu=0$ versus $\mathcal{M}_{0}: \mu=\mu_{1}$, which tests two point hypotheses; we refer to this case as the 'point null versus point alternative test'. The second test is $\mathcal{M}_{0}: \mu=0$ versus $\mathcal{M}_{0}: \mu \sim \mathcal{N}\left(0, \sigma_{1}^{2}\right)$ with $\sigma_{1}^{2}$ known; we refer to this case as the 'point null versus interval alternative test'. In what follows, we will consider each of these two tests in turn. In our derivations, we provide a proof for Assertion 1 for a fixed sample size $n$. For Assertion 2 we only provide a proof for a particular situation, namely, that the assertion holds after exactly one step of the optional stopping procedure. For a general mathematical proof of the adequacy of the calibration of Bayes factors under Bayesian optional stopping we again refer the reader to Hendriksen et al. (2020).

\section{Point null versus point alternative test}

Suppose data are independently normally distributed: $X_{i} \sim \mathcal{N}\left(\mu, \sigma^{2}\right)$ for $i=$ $1, \ldots, n$ and known variance $\sigma^{2}$. Our goal is to compare the predictive value of the following two models for parameter $\mu: \mathcal{M}_{0}: \mu=0$ and $\mathcal{M}_{1}: \mu=\mu_{1}$. The Bayes factor $B F_{10}$ is given as follows (see Theorem 1 in the supplemental material for a derivation):

$$
B F_{10}=\exp \left[\frac{n \mu_{1}\left(2 \bar{X}-\mu_{1}\right)}{2 \sigma^{2}}\right] .
$$

Working with logarithms simplifies the derivations, thus consider the following expression instead:

$$
\ln \left(B F_{10}\right)=\frac{n \mu_{1}\left(2 \bar{X}-\mu_{1}\right)}{2 \sigma^{2}} .
$$

We next derive the sampling distribution of $\ln \left(B F_{10}\right)$ under each model, $\mathcal{M}_{0}$ and $\mathcal{M}_{1}$, and then divide both densities and show that it results in the Bayes factor. This proves Assertion 1.

\subsection{Initial $n$ observations}

In general, the sampling distribution of $\bar{X}$ when the population mean is fixed (say, at $\mu_{X}$ ) is given by

$$
\bar{X} \sim \mathcal{N}\left(\mu_{X}, \frac{\sigma^{2}}{n}\right) .
$$

$\ln \left(B F_{10}\right)$ is a linear transformation of $\bar{X}$, thus the density of $\ln \left(B F_{10}\right)$ can be found by means of the change of variable theorem (see Lemma 2 in the supplemental material for a derivation):

$$
f_{\ln \left(B F_{10}\right)}(y)=\frac{\sigma}{\sqrt{n} \mu_{1}} \phi\left(\frac{\sigma}{\sqrt{n} \mu_{1}} y+\frac{\sqrt{n} \mu_{1}}{2 \sigma}-\frac{\sqrt{n}}{\sigma} \mu_{X}\right),
$$


where $y$ is a realization of $\ln \left(B F_{10}\right)$ and $\phi$ denotes the probability density function of the standard normal distribution. We therefore conclude that the sampling distribution of $\ln \left(B F_{10}\right)$ under $\mathcal{M}_{0}$ and $\mathcal{M}_{1}$ is given by Equation 6 for $\mu_{X}$ equal to 0 and $\mu_{1}$, respectively.

\subsubsection{Dividing the densities}

The final step consists of showing that $\frac{f_{\ln \left(B_{10}\right)}^{\mathcal{M}_{1}}(y)}{f_{\ln \left(B F_{10}\right)}^{\mathcal{M}_{0}}(y)}=\exp (y)$. Since $y$ is a realization of $\ln \left(B F_{10}\right), \exp (y)$ is the realization of $B F_{10}$ for the corresponding observed data, which concludes the proof of Assertion 1.

$$
\begin{aligned}
\frac{f_{\ln \left(B F_{10}\right)}^{\mathcal{M}_{1}}(y)}{f_{\ln \left(B F_{10}\right)}^{\mathcal{M}_{0}}(y)} & =\frac{\exp \left[-\frac{1}{2}\left(\frac{\sigma}{\sqrt{n} \mu_{1}} y-\frac{\sqrt{n} \mu_{1}}{2 \sigma}\right)^{2}\right]}{\exp \left[-\frac{1}{2}\left(\frac{\sigma}{\sqrt{n} \mu_{1}} y+\frac{\sqrt{n} \mu_{1}}{2 \sigma}\right)^{2}\right]} \\
& =\exp \left[-\frac{1}{2}(-4) \frac{\sigma}{\sqrt{n} \mu_{1}} y \frac{\sqrt{n} \mu_{1}}{2 \sigma}\right] \\
& =\exp (y) .
\end{aligned}
$$

3.2 Proceed with optional stopping

We need a rule to decide whether the evidence provided by the first $n$ observations is decisive (warranting the interruption of the optional stopping). As explained above, we set up the same rule as Rouder (2014): Stop if $B F_{10}>$ $B F_{U}=10\left(\right.$ retain $\left.\mathcal{M}_{1}\right)$ or $B F_{10}<B F_{L}=\frac{1}{10}\left(\right.$ retain $\mathcal{M}_{0}$ ), otherwise proceed sampling. Equation 4 allows reexpressing the decision rule in terms of the observed sample mean. Let $\bar{X}_{0}$ and $\bar{X}_{1}$ denote the sample mean values associated with $B F_{L}$ and $B F_{U}$, respectively. Solving Equation 4 with respect to $\bar{X}$ gives

$$
\bar{X}=\frac{1}{2} \mu_{1}+\frac{\sigma^{2}}{n \mu_{1}} \ln \left(B F_{10}\right) .
$$

We conclude that $\bar{X}_{0}=\frac{1}{2} \mu_{1}-\frac{\sigma^{2}}{n \mu_{1}} \ln (10)$ and $\bar{X}_{1}=\frac{1}{2} \mu_{1}+\frac{\sigma^{2}}{n \mu_{1}} \ln (10)$. The sequential testing procedure stops after the first $n$ observations when $\bar{X} \leq \bar{X}_{0}$ (retain $\mathcal{M}_{0}$ ) or $\bar{X} \geq \bar{X}_{1}$ (retain $\mathcal{M}_{1}$ ). The test is indecisive for all sample means in the interval $\mathcal{I}=\left(\bar{X}_{0}, \bar{X}_{1}\right)$.

In case $\bar{X} \in \mathcal{I}$, we need to collect more data and reassess the relative evidence between both models. Suppose $k(k \geq 1)$ more observations are collected. It is important to distinguish between the first $n$ observations (now fixed) from the $k$ new observations (to be sampled). We therefore express the sample mean value of the $(n+k)$ observations as follows:

$$
\bar{X}_{n+k}=\frac{n}{n+k}\left(\bar{X}_{n}+\frac{\sum_{i=n+1}^{n+k} X_{i}}{n}\right),
$$


where we now introduce the general notation $\bar{X}_{t}$ to refer to the sample mean based on the first $t$ observations. After the $k$ new observations are available, we use Equation 4 to recompute $\ln \left(B F_{10}\right)$ :

$$
\begin{aligned}
\ln \left(B F_{10}\right) & =\frac{(n+k) \mu_{1}\left(2 \bar{X}_{n+k}-\mu_{1}\right)}{2 \sigma^{2}} \\
& =\frac{(n+k) \mu_{1}}{2 \sigma^{2}}\left[\frac{2 n}{n+k}\left(\bar{X}_{n}+\frac{\sum_{i=n+1}^{n+k} X_{i}}{n}\right)-\mu_{1}\right] .
\end{aligned}
$$

The remaining of the proof consists of deriving the density of $\ln \left(B F_{10}\right)$ given by Equation 10 under each model, and then relate the ratio of both densities to the Bayes factor. We note that this is the distribution conditional on indecisive evidence after the first $n$ observations. We first derive the distribution of the sum $S=\bar{X}_{n}+\frac{\sum_{i=n+1}^{n+k} X_{i}}{n} ; \ln \left(B F_{10}\right)$ is simply a linear transformation of $S$.

Firstly, and again assuming that the true population mean is $\mu_{X}$ (a general unknown quantity), we observe that $\bar{X}_{n}$ now follows a truncated normal distribution:

$$
\bar{X}_{n} \sim \mathcal{N}_{T}\left(\mu_{X}, \frac{\sigma^{2}}{n} ; \mathcal{I}\right) .
$$

Moreover, from $X_{i} \sim \mathcal{N}\left(\mu_{X}, \sigma^{2}\right)$ it follows that

$$
\frac{\sum_{i=n+1}^{n+k} X_{i}}{n} \sim \mathcal{N}\left(\frac{k}{n} \mu_{X}, \frac{k}{n^{2}} \sigma^{2}\right) \text {. }
$$

Random variables $\bar{X}_{n}$ and $\frac{\sum_{i=n+1}^{n+k} X_{i}}{n}$ are independent, since sampling the $k$ new observations is independent from the sampling of the first $n$ observations. Hence, the distribution of $S$ is given by the convolution of both densities (e.g., Blitzstein and Hwang 2019, section 8.2). We derive the closed form expression of the density of $S$ in the supplemental material (see Theorem 3 for the general case, and Lemmas 3 and 4 for the particular cases under $\mathcal{M}_{0}$ and $\mathcal{M}_{1}$, resp.). Finally, the density of $\ln \left(B F_{10}\right)$ is found by means of the change of variable theorem (supplemental material, Lemma 5).

\subsubsection{Dividing the densities}

Finally, we show that the ratio of the densities of $\ln \left(B F_{10}\right)$ under either model, after $(n+k)$ observations, leads to the Bayes factor (supplemental material, Lemma 6).

\subsubsection{Unconditional distribution}

What we just established above is the closed-form expression of the distribution of $\ln \left(B F_{10}\right)$ under either model, conditional on an indecisive test result after the first $n$ observations. Theorem 4 in the supplemental material can now be used to ascertain that the Bayes factor is well calibrated overall, that is, after the initial $n$ observations (in case the first test was decisive) or after $(n+k)$ observations (in case the first test was indecisive). 


\subsection{Example 1}

We illustrate our derivations by means of an example. We use simulations as in Rouder (2014) and compare the approximate results from the simulations with our precise derivations. Let $n=10, k=5, \sigma=.3$, and $\mu_{1}=.1$. For each simulation (total $=100,000$ simulations), we followed this procedure:

1. Randomly draw $n$ observations from $\mathcal{N}\left(0, \sigma^{2}\right)$ (i.e., under $\left.\mathcal{M}_{0}\right)$. Compute $\bar{X}$ and $\ln \left(B F_{10}\right)$; compare with the corresponding exact distributions (Equations 5 and 6 with $\left.\mu_{X}=0\right)$.

2. Randomly draw $n$ observations from $\mathcal{N}\left(\mu_{1}, \sigma^{2}\right)$ (i.e., under $\left.\mathcal{M}_{1}\right)$. Compute $\bar{X}$ and $\ln \left(B F_{10}\right)$; compare with the corresponding exact distributions (Equations 5 and 6 with $\mu_{X}=\mu_{1}$ ).

3. Approximate the ratio of the densities of $\ln \left(B F_{10}\right)$ under either model and compare it to $B F_{10}$. The approximation is achieved by partitioning the support interval in small subintervals ${ }^{5}$ and then computing the ratio of the observed frequencies in each subinterval. This gives the observed (approximate) density ratio, which we compare to its exact counterpart (Equation 7).

4. Stop in case $B F_{10}$ is larger than 10 or smaller than $\frac{1}{10}$, otherwise: Collect $k$ more observations, recompute $\bar{X}$ and $\ln \left(B F_{10}\right)$ under each model, and approximate the ratio of the densities of $\ln \left(B F_{10}\right)$ under either model and compare it to $B F_{10}$.

Panels $\mathrm{A}$ and $\mathrm{B}$ of Figure 3 show the approximate distribution (histogram) and exact distribution (solid line) of $\bar{X}$ and $\ln \left(B F_{10}\right)$ under $\mathcal{M}_{0}$ and $\mathcal{M}_{1}$, after the initial $n$ observations. As can be seen, our exact distributions work as intended. In panel $\mathrm{C}$ we plot the approximate (points) and exact (solid line) density ratio. Our results imply that the line in panel $\mathrm{C}$ is the exponential function (see Equation 7). The conclusion is that, as proved, the ratio of frequencies of observed Bayes factor values under either model (as also done by Rouder 2014 ) is just an approximation to that very same nominal Bayes factor value.

Figures 4 and 5 show the distributions of $\bar{X}_{n}, \frac{\sum_{i=n+1}^{n+k} X_{i}}{n}$, and $S$ under $\mathcal{M}_{0}$ and $\mathcal{M}_{1}$, respectively. Figure 6 illustrates the most relevant result for the point null versus point alternative test considered here: After optional stopping, the ratio of the densities of the Bayes factor values under each model does indeed lead to the Bayes factor, as proved above.

\subsubsection{Probability of interesting events}

One added value of knowing the distribution of $B F_{10}$ under either model is that it allows quantifying the probability of interesting events. For example, by means of Equation 8 we know that the procedure is inconclusive after $n$ observations when $\bar{X}_{n}$ is in $\mathcal{I}=(-0.157,0.257)$. We can quantify the probability

\footnotetext{
5 Twenty-one subintervals of equal length were used. This number of subintervals provided enough accuracy for our purposes (each random variable is based on samples of 100,000 draws).
} 

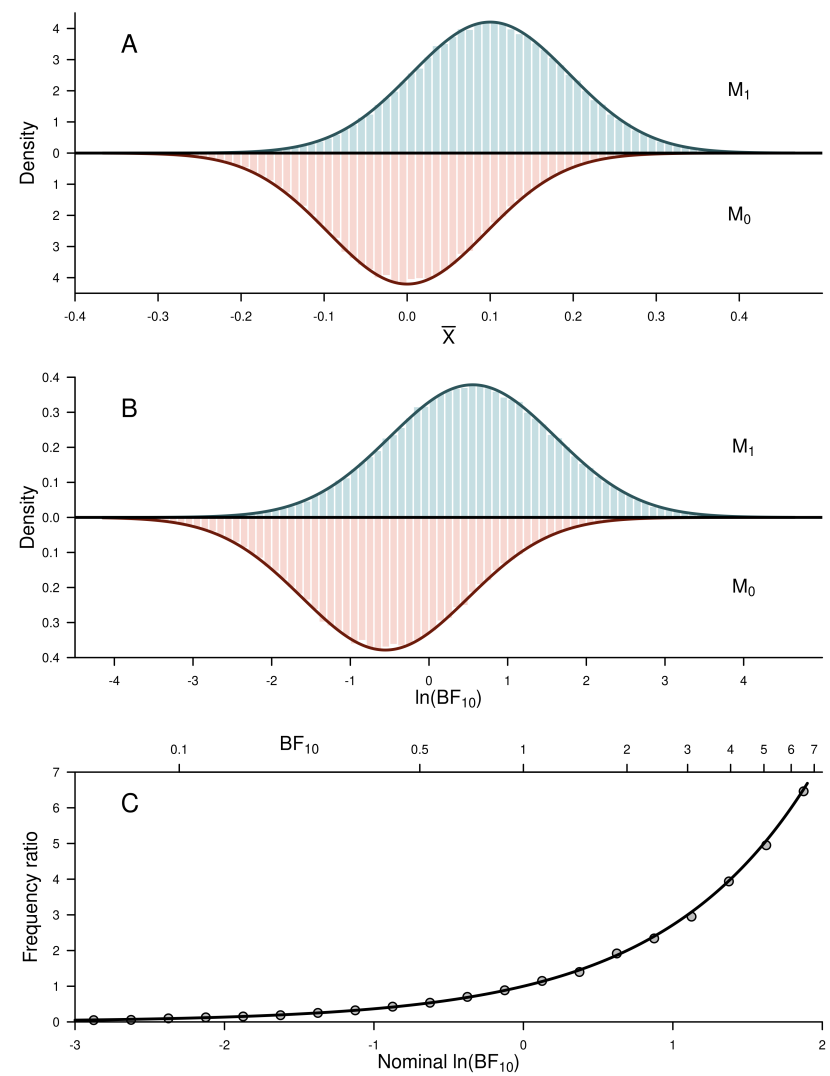

Fig. 3 Distribution of various random variables after $n$ observations, for the point null versus point alternative test. Panel $\mathrm{A}: \bar{X}$ under $\mathcal{M}_{0}$ (upper distribution; Equation 5 with $\mu_{X}=0$ ) and under $\mathcal{M}_{1}$ (inverted distribution; Equation 5 with $\left.\mu_{X}=\mu_{1}\right)$. Panel B: $\ln \left(B F_{10}\right)$ under $\mathcal{M}_{0}$ (upper distribution; Equation 6 with $\mu_{X}=0$ ) and under $\mathcal{M}_{1}$ (inverted distribution; Equation 6 with $\mu_{X}=\mu_{1}$ ). Panel C: Relation between the nominal and observed $\ln \left(B F_{10}\right)$ values based on approximation by simulation (points) and on the exact values (line) (Equation 7)

of each possible outcome (i.e., retain $\mathcal{M}_{0}$, retain $\mathcal{M}_{1}$, or inconclusive) under each model through either the distribution of $\bar{X}$ (Equation 5) or, equivalently, the distribution of $\ln \left(B F_{10}\right)$ (Equation 6 ); see Table 1 (top panel). Similarly, we can compute the overall probabilities of each event after the $k$ extra observations are collected (Table 1 , bottom panel). Because both hypotheses being tested are of the same type, the probabilities in Table 1 are symmetric (e.g., $p\left(\right.$ retain $\mathcal{M}_{0} \mid \mathcal{M}_{0}, n$ obs. $)=p\left(\right.$ retain $\mathcal{M}_{1} \mid \mathcal{M}_{1}, n$ obs. $\left.)=.049\right)$. It can be observed that, in this situation, the addition of $k$ extra observations increases the probability of making a correct decision. 

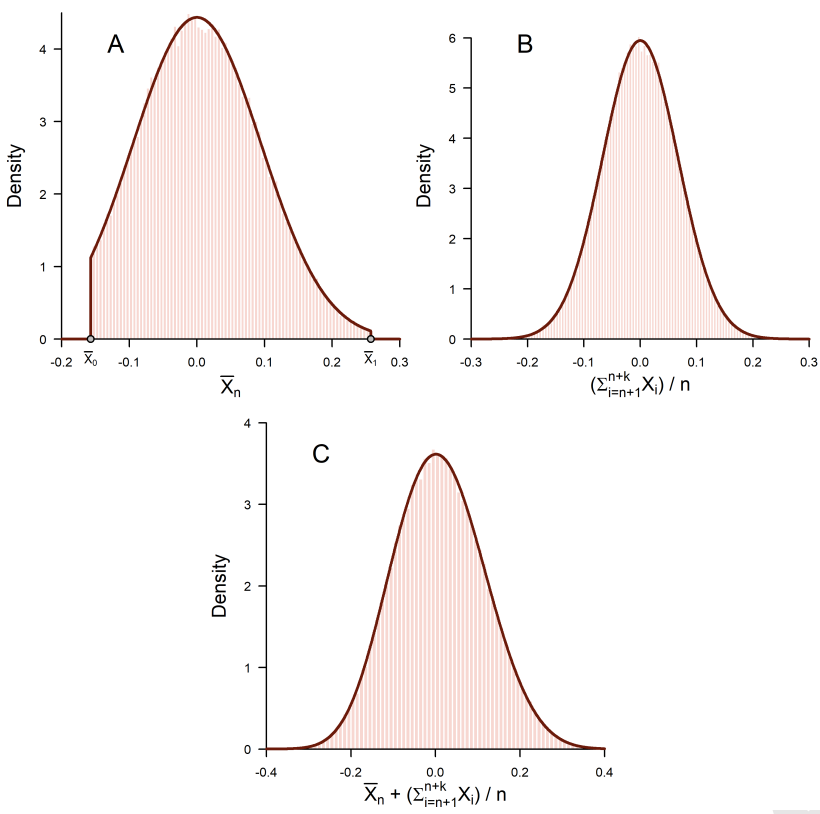

Fig. 4 Distribution of various random variables under $\mathcal{M}_{0}$ after $(n+k)$ observations, for the point null versus point alternative test. These random variables correspond to intermediate results that facilitate the proof of Assertion 2 (supplemental material, Lemma 6). Panel A: $\bar{X}_{n}$ (Equation 11 with $\left.\mu_{X}=0\right)$. Panel B: $\frac{\sum_{i=n+1}^{n+k} X_{i}}{n}$ (Equation 12 with $\mu_{X}=0$ ). Panel C: $S=\bar{X}_{n}+\frac{\sum_{i=n+1}^{n+k} X_{i}}{n}$ (supplemental material, Lemma 3).

Table 1 Point null versus point alternative test, Example 1 ( $n=10, k=5, \sigma=.3$, and $\mu_{1}=.1$ ): Probability of each decision after $n$ observations (top panel) and overall after $(n+k)$ observations (bottom panel), under either model.

\begin{tabular}{ccccc}
\hline After... & Model & $\begin{array}{c}\text { Stop and } \\
\text { retain } \mathcal{M}_{0}\end{array}$ & $\begin{array}{c}\text { Proceed } \\
\text { data collection }\end{array}$ & $\begin{array}{c}\text { Stop and } \\
\text { retain } \mathcal{M}_{1}\end{array}$ \\
\hline$n$ & $\mathcal{M}_{0}$ & .049 & .948 & .003 \\
observations & $\mathcal{M}_{1}$ & .003 & .948 & .049 \\
\hline$n+k$ & $\mathcal{M}_{0}$ & .137 & .854 & .009 \\
observations & $\mathcal{M}_{1}$ & .009 & .854 & .137 \\
\hline
\end{tabular}

\section{Point null versus interval alternative test}

We now look at a different alternative model for $\mu$ and consider testing $\mathcal{M}_{0}$ : $\mu=0$ versus $\mathcal{M}_{1}: \mu \sim \mathcal{N}\left(0, \sigma_{1}^{2}\right)$, with $\sigma_{1}$ known. This test has been studied before (e.g., Berger and Delampady 1987; Berger and Pericchi 2001; Rouder 2014; Rouder et al. 2018). The Bayes factor $B F_{10}$ is given by (for a derivation see, e.g., Tendeiro and Kiers 2019, Appendix B):

$$
B F_{10}=\frac{\sigma}{\sqrt{\sigma^{2}+n \sigma_{1}^{2}}} \exp \left[\frac{n^{2} \sigma_{1}^{2} \bar{X}^{2}}{2 \sigma^{2}\left(\sigma^{2}+n \sigma_{1}^{2}\right)}\right],
$$



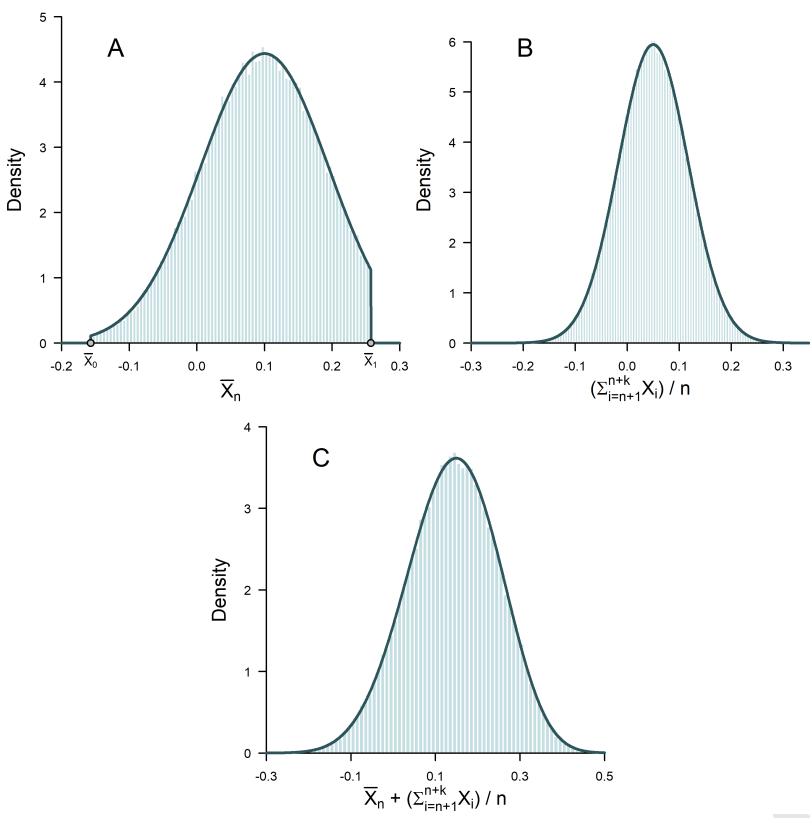

Fig. 5 Distributions of various random variables under $\mathcal{M}_{1}$ after $(n+k)$ observations, for the point null versus point alternative test. These random variables correspond to intermediate results that facilitate the proof of Assertion 2 (supplemental material, Lemma 6). Panel A: $\bar{X}_{n}$ (Equation 11 with $\mu_{X}=\mu_{1}$ ). Panel B: $\frac{\sum_{i=n+1}^{n+k} X_{i}}{n}$ (Equation 12 with $\mu_{X}=\mu_{1}$ ). Panel C: $S=\bar{X}_{n}+\frac{\sum_{i=n+1}^{n+k} X_{i}}{n}$ (supplemental material, Lemma 4).

or in terms of logarithms,

$$
\ln \left(B F_{10}\right)=\lambda+\frac{n^{2} \sigma_{1}^{2} \bar{X}^{2}}{2 \sigma^{2}\left(\sigma^{2}+n \sigma_{1}^{2}\right)}
$$

where $\lambda=\ln \left(\frac{\sigma}{\sqrt{\sigma^{2}+n \sigma_{1}^{2}}}\right)$ does not depend on the observed data.

We will follow a similar strategy as for the point null versus point alternative test. First, we derive the sampling distribution of $\ln \left(B F_{10}\right)$ under each model after the first $n$ observations, and show that the ratio of both densities results in the Bayes factor. Second, we collect $k$ more observations for the cases where evidence is inconclusive and reassess the distributions of $\ln \left(B F_{10}\right)$ under each model. Once more, we will establish that the ratio of both densities results in the Bayes factor. 

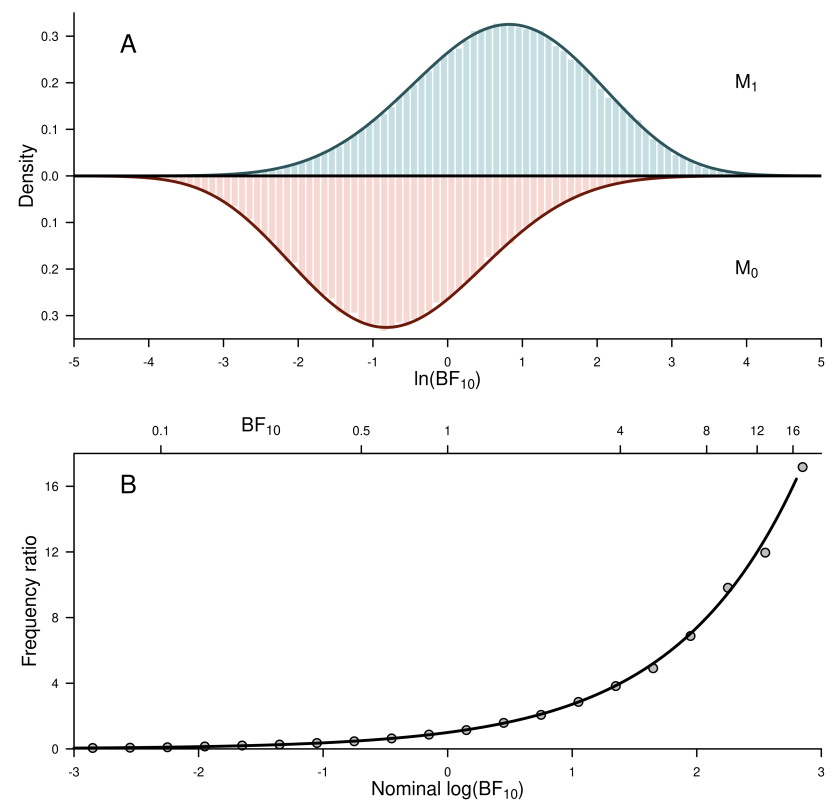

Fig. 6 Visualization of Assertion 2 for the point null versus point alternative test. Panel A: $\ln \left(B F_{10}\right)$ under $\mathcal{M}_{0}$ (upper distribution) and under $\mathcal{M}_{1}$ (lower distribution) (supplemental material, Lemma 5). Panel B: Relation between the nominal and observed $\ln \left(B F_{10}\right)$ values based on approximation by simulation (points) and on the exact values (line) (supplemental material, Lemma 6$)$, after $(n+k)$ observations for the point null versus point alternative test.

\subsection{Under $\mathcal{M}_{0}$}

When $\mu=0$, the sampling distribution of $\bar{X}$ is given by $\bar{X} \mid \mathcal{M}_{0} \sim \mathcal{N}\left(0, \frac{\sigma^{2}}{n}\right)$ (Equation 5 with $\mu_{X}=0$ ). It then follows that $\frac{\bar{X}-0}{\sigma / \sqrt{n}} \sim \mathcal{N}(0,1)$ and $\left(\frac{\bar{X}-0}{\sigma / \sqrt{n}}\right)^{2}=$ $\frac{n \bar{X}^{2}}{\sigma^{2}} \sim \chi_{1}^{2}$. Finally, observe that $\ln \left(B F_{10}\right)$ (Equation 14) is a linear function of $\frac{n \bar{X}^{2}}{\sigma^{2}}$, thus the distribution $\ln \left(B F_{10}\right)$ can be found by the change of variable theorem (see Lemma 7 in the supplemental material, with $\sigma_{X}^{2}=\frac{\sigma^{2}}{n}$ ):

$$
f_{\ln \left(B F_{10}\right)}^{\mathcal{M}_{0}}(y)=\frac{2\left(\sigma^{2}+n \sigma_{1}^{2}\right)}{n \sigma_{1}^{2}} f_{\chi_{1}^{2}}\left[\frac{2\left(\sigma^{2}+n \sigma_{1}^{2}\right)(y-\lambda)}{n \sigma_{1}^{2}}\right] \text {, for } y>\lambda
$$

\subsection{Under $\mathcal{M}_{1}$}

The sampling distribution of $\bar{X}$ is given by

$$
p\left(\bar{X} \mid \mathcal{M}_{1}\right)=\int_{-\infty}^{\infty} p\left(\bar{X} \mid \mu, \mathcal{M}_{1}\right) p\left(\mu \mid \mathcal{M}_{1}\right) d \mu
$$


where $\bar{X} \mid \mu, \mathcal{M}_{1} \sim \mathcal{N}\left(\mu, \frac{\sigma^{2}}{n}\right)$ and $\mu \mid \mathcal{M}_{1} \sim \mathcal{N}\left(0, \sigma_{1}^{2}\right)$. In the supplemental material (Lemma 8 ) we solve the integral and conclude that

$$
\bar{X} \mid \mathcal{M}_{1} \sim \mathcal{N}\left(0, \frac{\sigma^{2}}{n}+\sigma_{1}^{2}\right)
$$

It then follows that $\frac{\bar{X}}{\sqrt{\frac{\sigma^{2}}{n}+\sigma_{1}^{2}}} \sim \mathcal{N}(0,1)$ and $\left(\frac{\bar{X}}{\sqrt{\frac{\sigma^{2}}{n}+\sigma_{1}^{2}}}\right)^{2}=\frac{n \bar{X}^{2}}{\sigma^{2}+n \sigma_{1}^{2}} \sim \chi_{1}^{2}$. Again, observe that $\ln \left(B F_{10}\right)$ (Equation 14) can be expressed as a linear function of $\frac{n \bar{X}^{2}}{\sigma^{2}+n \sigma_{1}^{2}}$, thus the distribution $\ln \left(B F_{10}\right)$ can be found by the change of variable theorem (see Lemma 7 in the supplemental material, with $\sigma_{X}^{2}=$ $\left.\frac{\sigma^{2}}{n}+\sigma_{1}^{2}\right)$ :

$$
f_{\ln \left(B F_{10}\right)}^{\mathcal{M}_{1}}(y)=\frac{2 \sigma^{2}}{n \sigma_{1}^{2}} f_{\chi_{1}^{2}}\left[\frac{2 \sigma^{2}(y-\lambda)}{n \sigma_{1}^{2}}\right], \text { for } y>\lambda
$$

4.3 Dividing the densities

As a final step, we show that $\frac{f_{\ln \left(B F_{10}\right)}^{\mathcal{M}_{1}}(y)}{f_{\ln \left(B F_{10}\right)}^{\mathcal{M}_{0}}(y)}=B F_{10}$, that is, the realization of $B F_{10}$ for the corresponding observed data (supplemental material, Lemma 9).

\subsection{Proceed with optional stopping}

More data are collected in case evidence from the first $n$ observations is indecisive. Equation 14 allows reexpressing the decision rule in terms of the observed sample mean; solving Equation 14 with respect to $\bar{X}$ gives

$$
\begin{aligned}
\bar{X}^{2} & =\left[\ln \left(B F_{10}\right)-\lambda\right] \frac{2 \sigma^{2}\left(\sigma^{2}+n \sigma_{1}^{2}\right)}{n^{2} \sigma_{1}^{2}} \\
\bar{X} & = \pm \frac{\sigma}{n \sigma_{1}} \sqrt{2\left(\sigma^{2}+n \sigma_{1}^{2}\right)\left[\ln \left(B F_{10}\right)-\lambda\right]},
\end{aligned}
$$

under the constraint that $\lambda<\ln \left(B F_{10}\right)$. Let $\bar{X}_{0}$ and $\bar{X}_{1}$ denote the value of $\bar{X}$ when $B F_{10}=B F_{L}=\frac{1}{10}$ and $B F_{10}=B F_{U}=10$, respectively. Observe that $\bar{X}_{1}$ always exists because $\frac{\sigma}{\sqrt{\sigma^{2}+n \sigma_{1}^{2}}}<1<10=B F_{10}$ and so $\lambda=\ln \left(\frac{\sigma}{\sqrt{\sigma^{2}+n \sigma_{1}^{2}}}\right)<\ln \left(B F_{10}\right) . \bar{X}_{0}$ exists if $\lambda<\ln \left(B F_{10}\right)=-\ln (10)$; this is possible when small enough absolute values of $\bar{X}$ are associated to values of $B F_{10}$ smaller than $\frac{1}{10}$, allowing to draw enough support for $\mathcal{M}_{0}$ and thus interrupting the optional stopping process. When $-\ln (10)<\lambda$ then even $\bar{X}=0$ is associated to a value of $B F_{10}$ not below $\frac{1}{10}$, which means that it is impossible to draw enough support for $\mathcal{M}_{0}$ after $n$ observations. In this case we set $\bar{X}_{0}=0$, which is the limiting case of the first situation. Figure 7 shows the 

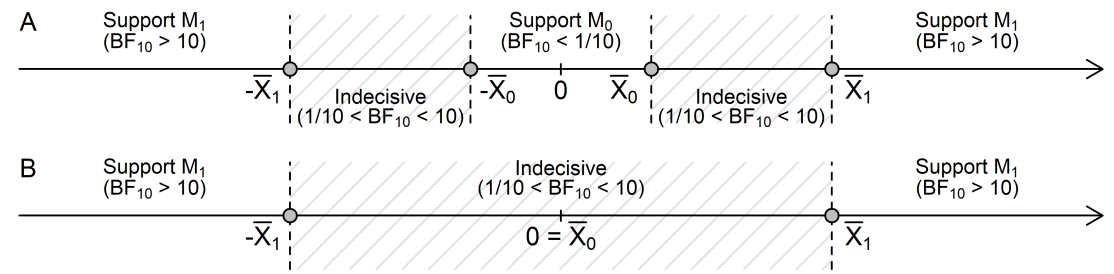

Fig. 7 Data evidence for either model after assessing the first batch of $n$ observations, for the point null versus interval alternative test. Panel A: The case when $\lambda<-\ln (10)$. Panel B: The case when $-\ln (10)<\lambda$.

two possible situations that may arise. In particular, the test after the first $n$ observations is indecisive when $\bar{X} \in \mathcal{J}=\left(-\bar{X}_{1},-\bar{X}_{0}\right) \cup\left(\bar{X}_{0}, \bar{X}_{1}\right)$.

In case evidence is indecisive after the initial $n$ observations, then the optional stopping procedure continues: Collect $k$ more observations and reassess the evidence by means of the Bayes factor:

$$
\begin{aligned}
\ln \left(B F_{10}\right) & =\lambda+\frac{(n+k)^{2} \sigma_{1}^{2} \bar{X}_{n+k}^{2}}{2 \sigma^{2}\left[\sigma^{2}+(n+k) \sigma_{1}^{2}\right]} \\
& =\alpha+\beta\left(\bar{X}_{n}+\frac{\sum_{i=n+1}^{n+k} X_{i}}{n}\right)^{2},
\end{aligned}
$$

where $\alpha=\ln \left[\frac{\sigma}{\sqrt{\sigma^{2}+(n+k) \sigma_{1}^{2}}}\right]$ and $\beta=\frac{n^{2} \sigma_{1}^{2}}{2 \sigma^{2}\left[\sigma^{2}+(n+k) \sigma_{1}^{2}\right]}$ do not depend on the observed data, and Equation 9 was used to decompose $\bar{X}_{n+k}$. In what follows, we derive the distribution of $\ln \left(B F_{10}\right)$ given by Equation 19 under each model, and then relate the ratio of both densities to the Bayes factor.

\subsubsection{Under $\mathcal{M}_{0}$}

In this case, $\bar{X}_{n}$ follows a truncated normal distribution:

$$
\bar{X}_{n} \mid \mathcal{M}_{0} \sim \mathcal{N}_{T}\left(0, \frac{\sigma^{2}}{n} ; \mathcal{J}\right),
$$

where the support for the truncation is the union of two intervals. Moreover, $\frac{\sum_{i=n+1}^{n+k} X_{i}}{n}$ is normally distributed (Equation 12 with $\mu_{X}=0$ ). Therefore, the distribution of the sum, $S=\bar{X}_{n}+\frac{\sum_{i=n+1}^{n+k} X_{i}}{n}$, is given by the convolution of both densities; the closed-form expression for this density is given in the supplemental material (Lemma 11). Finally, observe that $\ln \left(B F_{10}\right)$ in Equation 19 is a linear function of the square of $S$. Hence, the density of $\ln \left(B F_{10}\right)$ can be found in two steps: First find the density of $S^{2}$ (supplemental material, Lemma 13) and then find the density of the linear transformation of $S^{2}$ (supplemental material, Lemma 14). 


\subsubsection{Under $\mathcal{M}_{1}$}

The distribution of the summation in Equation 19 is more difficult to derive under $\mathcal{M}_{1}$. The reason is that the sampling distribution of a new observation is affected by conditioning on an indecisive test result after the initial $n$ observations. Observe that, by Bayes' rule,

$$
p\left(\mu \mid \bar{X}_{n} \in \mathcal{J}\right)=\frac{p(\mu) p\left(\bar{X}_{n} \in \mathcal{J} \mid \mu\right)}{p\left(\bar{X}_{n} \in \mathcal{J}\right)},
$$

where $\mu \sim \mathcal{N}\left(0, \sigma_{1}^{2}\right), \bar{X}_{n} \mid \mu \sim \mathcal{N}\left(\mu, \frac{\sigma^{2}}{n}\right)$, and $p\left(\bar{X}_{n} \in \mathcal{J}\right)=\int_{\mathbb{R}} p(\mu) p\left(\bar{X}_{n} \in\right.$ $\mathcal{J} \mid \mu) d \mu$. We derived the density of $\mu \mid \bar{X}_{n} \in \mathcal{J}$ (supplemental material, Lemma 15). This is the distribution of $\mu$ after the first optional stopping step, that is, after an inconclusive test result based on the first $n$ data points.

Now consider the summation in Equation 19, but conditional on $\mu$. We have that $\bar{X}_{n} \mid \mu, \mathcal{M}_{1} \sim \mathcal{N}_{T}\left(\mu, \frac{\sigma^{2}}{n} ; \mathcal{J}\right)$ and $\frac{\sum_{i=n+1}^{n+k} X_{i}}{n} \mid \mu, \mathcal{M}_{1} \sim \mathcal{N}\left(\frac{k}{n} \mu, \frac{k}{n^{2}} \sigma^{2}\right)$. The sum of these two independent conditional distributions, say $S\left|\mu=\bar{X}_{n}\right| \mu+$ $\frac{\sum_{i=n+1}^{n+k} X_{i}}{n} \mid \mu$, is the convolution of both densities; the distribution is given in the supplemental material, Lemma 16. To find the density of the unconditional sum we need to evaluate $f_{S}(s)=\int_{\mathbb{R}} p(S=s \mid \mu) p\left(\mu \mid \bar{X}_{n} \in \mathcal{J}\right) d \mu$. The exact closed form of this integral has evaded us. Instead, we resourced to numerical integration to approximate the integral. We next proceeded using numerical integration to approximate the density of $S^{2}$ and the density of $\ln \left(B F_{10}\right)$ as a linear function of $S^{2}$. Finally, we numerically divided the densities of $\ln \left(B F_{10}\right)$ (under $\mathcal{M}_{1}$ and $\mathcal{M}_{0}$ ) and compared the result with the Bayes factor. Also here, Theorem 4 in the supplemental material ascertains that the previous result holds overall (i.e., after the initial $n$ observations or after $(n+k)$ observations, depending on the first test result). The example below illustrates what is happening.

\subsection{Example 2}

Let $n=10, k=5, \sigma=.3$, and $\sigma_{1}=1.1$. For each simulation (total $=100,000$ simulations), we followed this procedure:

1. Randomly draw $n$ observations from $\mathcal{N}\left(0, \sigma^{2}\right)$ (i.e., under $\left.\mathcal{M}_{0}\right)$. Compute $\ln \left(B F_{10}\right)$ and compare with the exact distribution (Equation 15).

2. Sample one $\mu$ value from $\mathcal{N}\left(0, \sigma_{1}^{2}\right)$. Randomly draw $n$ observations from $\mathcal{N}\left(\mu, \sigma^{2}\right)$ (i.e., under $\left.\mathcal{M}_{1}\right)$. Compute $\ln \left(B F_{10}\right)$ and compare with the exact distribution (Equation 17).

3. Approximate the ratio of the densities of $\ln \left(B F_{10}\right)$ under either model and compare it to $B F_{10}$ and compare to its exact counterpart (supplemental material, Lemma 9). 
Table 2 Point null versus interval alternative test, Example $2(n=10, k=5, \sigma=.3$, and $\sigma_{1}=1.1$ ): Probability of each decision after $n$ observations (top panel) and overall after $(n+k)$ observations (bottom panel), under either model.

\begin{tabular}{ccccc}
\hline After... & Model & $\begin{array}{c}\text { Stop and } \\
\text { retain } \mathcal{M}_{0}\end{array}$ & $\begin{array}{c}\text { Proceed } \\
\text { data collection }\end{array}$ & $\begin{array}{c}\text { Stop and } \\
\text { retain } \mathcal{M}_{1}\end{array}$ \\
\hline$n$ & $\mathcal{M}_{0}$ & .420 & .578 & .002 \\
observations & $\mathcal{M}_{1}$ & .038 & .172 & .790 \\
\hline$n+k$ & $\mathcal{M}_{0}$ & .677 & .320 & .003 \\
observations & $\mathcal{M}_{1}$ & .059 & .113 & .828 \\
\hline
\end{tabular}

4. Stop in case $B F_{10}$ is larger than 10 or smaller than $\frac{1}{10}$, otherwise: Collect $k$ more observations, recompute $\ln \left(B F_{10}\right)$ under each model, and approximate the ratio of the densities of $\ln \left(B F_{10}\right)$ under either model and compare it to $B F_{10}$.

Figure 8 shows the approximate distribution (histogram) and exact distribution (solid line) of $\bar{X}$ and $\ln \left(B F_{10}\right)$ under $\mathcal{M}_{0}$ and $\mathcal{M}_{1}$, as well as the density ratio of the Bayes factors under either model, after the initial $n$ observations. Figure 9 shows the distributions of $\bar{X}_{n}, \frac{\sum_{i=n+1}^{n+k} X_{i}}{n}, S$, and $S^{2}$ under $\mathcal{M}_{0}$, after $(n+k)$ observations. Figure 10 shows the distributions of $\mu \mid \bar{X}_{n} \in \mathcal{J}$ (Equation 21), $\bar{X}_{n} \mid \mu=.1$ (as one example of such a conditional distribution), $\frac{\sum_{i=n+1}^{n+k} X_{i}}{n}|\mu=.1, S| \mu=.1$, and $S$ under $\mathcal{M}_{1}$. Finally, Figure 11 shows that the ratio of the densities of the Bayes factor values under each model is given by the Bayes factor.

In the current setting, the sequential testing procedure is inconclusive after the initial $n$ observations when $\bar{X}_{n} \in \mathcal{J}=(-.294,-.052) \cup(.052, .294)$ (Equation 18). As before, we can quantify the probability of retaining $\mathcal{M}_{0}$, retaining $\mathcal{M}_{1}$, or proceeding data collection under each model, after $n$ and after $(n+k)$ observations (see Table 2$)$. The conclusion is similar to that from the previous example, namely, increasing the sample size increased the probability of finding support for the correct model.

\section{Discussion}

In this paper we worked out two examples introduced by Rouder (2014) that were originally used to motivate why Bayes factors are well calibrated under Bayesian optional stopping (recently dubbed 'Sequential Bayes Factor' (SBF) by Schönbrodt et al. 2017). A completely general proof of this result is already offered by Hendriksen et al. (2020, Corollary 3), but the technical nature of this paper makes it rather inaccessible to less math-savvy readers. Our results offer a less ambitious but perhaps more insightful approach to understanding the findings presented in Rouder (2014), which were entirely simulation-based. Our contribution leaves us one step closer to understanding the Bayes factor and its properties under optional stopping. 

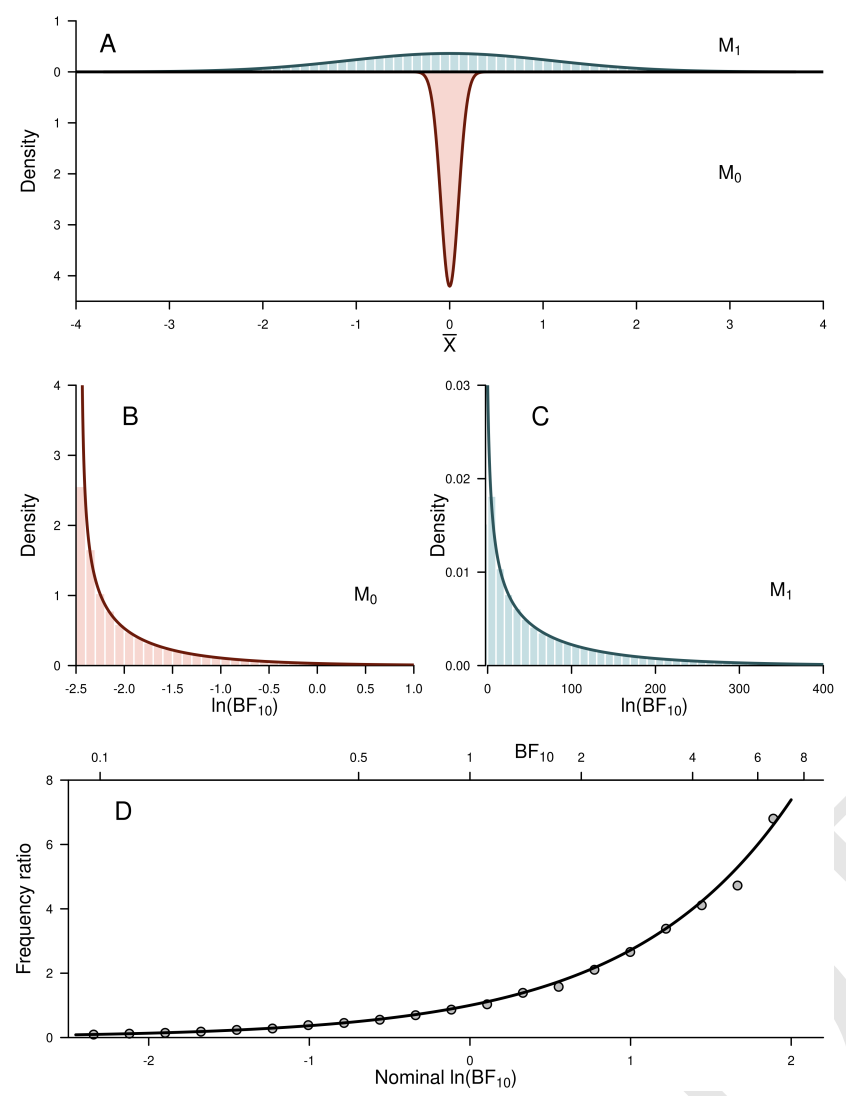

Fig. 8 Distribution of various random variables after $n$ observations, for the point null versus interval alternative test. Panel $\mathrm{A}: \bar{X}$ under $\mathcal{M}_{0}$ (upper distribution; $\mathcal{N}\left(0, \frac{\sigma^{2}}{n}\right)$ ) and under $\mathcal{M}_{1}$ (inverted distribution; Equation 16). Panel B: $\ln \left(B F_{10}\right)$ under $\mathcal{M}_{0}$ (Equation 15). Panel C: $\ln \left(B F_{10}\right)$ under $\mathcal{M}_{1}$ (Equation 17). Panel D: Relation between the nominal and observed $\ln \left(B F_{10}\right)$ values based on approximation by simulation (points) and on the exact values (line) (supplemental material, Lemma 9).

Our results are limited in various ways. Firstly, we focused exclusively on the two tests used by Rouder (2014). Secondly, our approach is not easily generalizable to more complex cases. We note that we could not fully work out the last part of the mathematical derivation in the point null versus interval alternative case. Thirdly, our theoretical results apply to the first and second batches of data. If, as is often the case, the evidence is still inconclusive after the second data batch is taken into account, then the optional stopping procedure should be able to proceed. Our proof does not provide the full answer in this case. This could be the goal of a future extension of our results.

Our paper attempts to build a bridge between the practical simulation results offered by Rouder (2014), and the theoretical proofs provided by Hendriksen et al. (2020). We generalize Rouder's simulation results such that they 

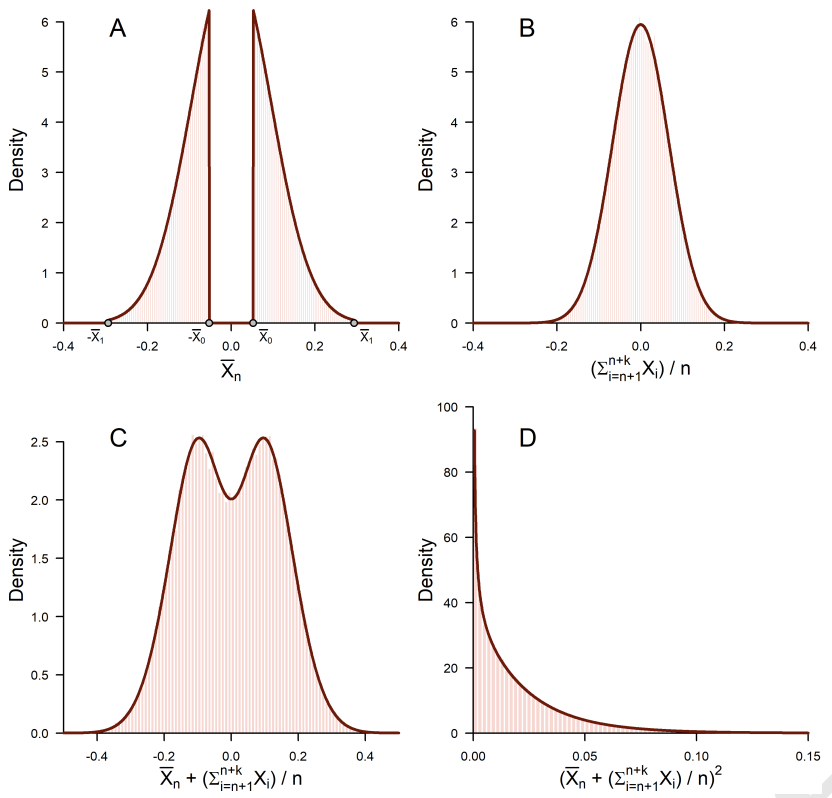

Fig. 9 Distribution of various random variables under $\mathcal{M}_{0}$ after $(n+k)$ observations, for the point null versus interval alternative test. Panel A: $\bar{X}_{n}$ (Equation 20). Panel B: $\frac{\sum_{i=n+1}^{n+k} X_{i}}{n}$ (Equation 12 with $\mu_{X}=0$ ). Panel C: $S=\bar{X}_{n}+\frac{\sum_{i=n+1}^{n+k} X_{i}}{n}$ (supplemental material, Lemma 11). Panel D: $S^{2}$ (supplemental material, Lemma 13).

do not rely on specific values and present them in a way that are, hopefully, digestible to social scientists.

\section{Open Practices Statement}

The $\mathrm{R}$ scripts including the code required to reproduce all computations and figures available in this paper are available at https://osf.io/5z92h/. No experiments were conducted and therefore preregistered.

\section{References}

Anscombe, F. J. (1954). Fixed-Sample-Size Analysis of Sequential Observations. Biometrics, 10(1):89-100.

Armitage, P. (1960). Sequential Medical Trials. Thomas, Springfield.

Armitage, P., McPherson, C. K., and Rowe, B. C. (1969). Repeated Significance Tests on Accumulating Data. Journal of the Royal Statistical Society. Series A (General), $132(2): 235-244$.

Berger, J. O. and Delampady, M. (1987). Testing precise hypotheses. Statistical Science, $2: 317-352$. 

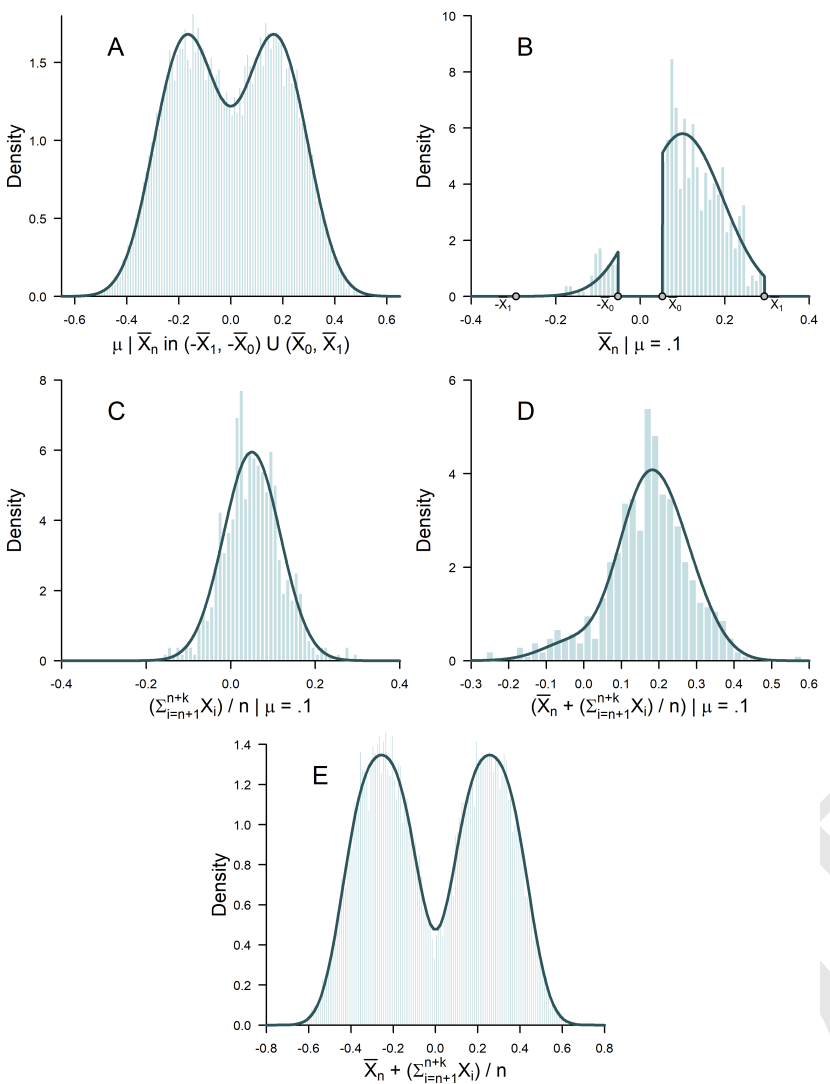

Fig. 10 Distributions of various random variables under $\mathcal{M}_{1}$ after $(n+k)$ observations, for the point null versus interval alternative test. Panel A: $\mu \mid \bar{X}_{n} \in \mathcal{J}$ (Equation 21). Panel B: $\bar{X}_{n} \mid \mu=.1\left(\mathcal{N}_{T}\left(\mu, \frac{\sigma^{2}}{n} ; \mathcal{J}\right)\right)$. Panel C: $\frac{\sum_{i=n+1}^{n+k} X_{i}}{n} \mid \mu=.1\left(\mathcal{N}\left(\frac{k}{n} \mu, \frac{k}{n^{2}} \frac{\sigma^{2}}{n}\right)\right)$. Panel D: $S=\bar{X}_{n}+\frac{\sum_{i=n+1}^{n+k} X_{i}}{n} \mid \mu=.1$ (supplemental material, Lemma 16). Panel E: $S$, by means of numerical integration.

Berger, J. O. and Pericchi, L. R. (2001). Objective Bayesian methods for model selection: Introduction and comparison. In Model Selection, pages 135-207. Institute of Mathematical Statistics Lecture Notes - Monograph Series, Beachwood.

Berger, J. O. and Wolpert, R. L. (1988). The Likelihood Principle. Institute of Mathematical Statistics, Hayward (CA).

Blitzstein, J. K. and Hwang, J. (2019). Introduction to Probability, Second Edition. Chapman and Hall/CRC, Boca Raton, second edition.

Botella, J., Ximénez, C., Revuelta, J., and Suero, M. (2006). Optimization of sample size in controlled experiments: The CLAST rule. Behavior Research Methods, 38(1):65-76.

de Heide, R. and Grünwald, P. D. (2017). Why optional stopping is a problem for Bayesians. arXiv:1708.08278 [math, stat].

Deng, A., Lu, J., and Chen, S. (2016). Continuous Monitoring of A/B Tests without Pain: Optional Stopping in Bayesian Testing. In 2016 IEEE International Conference on Data Science and Advanced Analytics (DSAA), pages 243-252. 

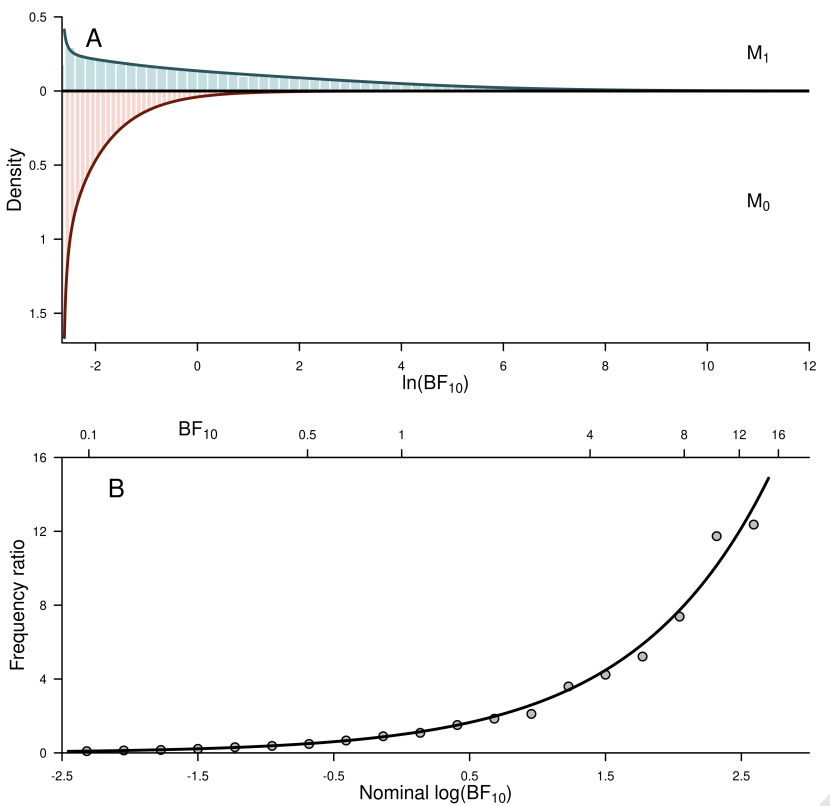

Fig. 11 Visualization of Assertion 2 for the point null versus interval alternative test. Panel A: $\ln \left(B F_{10}\right)$ under $\mathcal{M}_{0}$ (upper distribution; supplemental material, Lemma 14) and under $\mathcal{M}_{1}$ (lower distribution, by means of numerical integration). Panel B: Relation between the nominal and observed $\ln \left(B F_{10}\right)$ values based on approximation by simulation (points) and on the exact values (line), after $(n+k)$ observations for the point null versus interval alternative test.

Edwards, W., Lindman, H., and Savage, L. J. (1963). Bayesian statistical inference for psychological research. Psychological Review, 70:193-242.

Etz, A., Haaf, J. M., Rouder, J. N., and Vandekerckhove, J. (2018). Bayesian Inference and Testing Any Hypothesis You Can Specify. Advances in Methods and Practices in Psychological Science, 1(2):281-295.

Fitts, D. A. (2010). Improved stopping rules for the design of efficient small-sample experiments in biomedical and biobehavioral research. Behavior Research Methods, 42(1):3-22.

Frick, R. W. (1998). A better stopping rule for conventional statistical tests. Behavior Research Methods, Instruments, \& Computers, 30(4):690-697.

Hendriksen, A., de Heide, R., and Grünwald, P. (2020). Optional Stopping with Bayes Factors: A Categorization and Extension of Folklore Results, with an Application to Invariant Situations. Bayesian Analysis.

Jeffreys, H. (1961). Theory of Probability. Oxford University Press, Oxford, third edition. Jennison, C. and Turnbull, B. W. (1990). Statistical Approaches to Interim Monitoring of Medical Trials: A Review and Commentary. Statistical Science, 5(3):299-317.

Jennison, C. and Turnbull, B. W. (1999). Group Sequential Methods with Applications to Clinical Trials. Chapman and Hall/CRC, Boca Raton, 1 edition edition.

John, L. K., Loewenstein, G., and Prelec, D. (2012). Measuring the Prevalence of Questionable Research Practices With Incentives for Truth Telling. Psychological Science, 23(5):524-532.

Kadane, J. B., Schervish, M. J., and Seidenfeld, T. (1996). Reasoning to a Foregone Conclusion. Journal of the American Statistical Association, 91(435):1228-1235.

Kass, R. E. and Raftery, A. E. (1995). Bayes factors. Journal of the American Statistical Association, 90:773-795. 
Lakens, D. (2014). Performing high-powered studies efficiently with sequential analyses: Sequential analyses. European Journal of Social Psychology, 44(7):701-710.

Lindley, D. V. (1957). A statistical paradox. Biometrika, 44(1-2):187-192.

Matzke, D., Nieuwenhuis, S., van Rijn, H., Slagter, H. A., van der Molen, M. W., and Wagenmakers, E.-J. (2015). The effect of horizontal eye movements on free recall: A preregistered adversarial collaboration. Journal of Experimental Psychology: General, 144(1):e1-e15.

Nosek, B. A., Ebersole, C. R., DeHaven, A. C., and Mellor, D. T. (2018). The preregistration revolution. Proceedings of the National Academy of Sciences, 115(11):2600-2606.

Nosek, B. A. and Lakens, D. (2014). Registered reports: A method to increase the credibility of published results. Social Psychology, 45(3):137-141.

Pocock, S. J. (1983). Clinical Trials: A Practical Approach. John Wiley \& Sons, Chichester West Sussex ; New York.

Rouder, J. N. (2014). Optional stopping: No problem for Bayesians. Psychonomic Bulletin G6 Review, 21(2):301-308.

Rouder, J. N. and Haaf, J. M. (2019). Optional Stopping and the Interpretation of The Bayes Factor. Preprint, PsyArXiv.

Rouder, J. N., Haaf, J. M., and Vandekerckhove, J. (2018). Bayesian inference for psychology, part IV: Parameter estimation and Bayes factors. Psychonomic Bulletin \& Review, 25:102-113.

Sanborn, A. N. and Hills, T. T. (2014). The frequentist implications of optional stopping on Bayesian hypothesis tests. Psychonomic Bulletin \& Review, 21(2):283-300.

Sanborn, A. N., Hills, T. T., Dougherty, M. R., Thomas, R. P., Yu, E. C., and Sprenger, A. M. (2014). Reply to Rouder (2014): Good frequentist properties raise confidence. Psychonomic Bulletin \& Review, 21(2):309-311.

Schönbrodt, F. D. and Wagenmakers, E.-J. (2018). Bayes factor design analysis: Planning for compelling evidence. Psychonomic Bulletin \& Review, 25(1):128-142.

Schönbrodt, F. D., Wagenmakers, E.-J., Zehetleitner, M., and Perugini, M. (2017). Sequential hypothesis testing with Bayes factors: Efficiently testing mean differences. Psychological Methods, 22(2):322-339.

Simmons, J. P., Nelson, L. D., and Simonsohn, U. (2011). False-Positive Psychology: Undisclosed Flexibility in Data Collection and Analysis Allows Presenting Anything as Significant. Psychological Science, 22(11):1359-1366.

Tendeiro, J. N. and Kiers, H. A. L. (2019). A review of issues about null hypothesis Bayesian testing. Psychological Methods.

van de Schoot, R., Winter, S. D., Ryan, O., Zondervan-Zwijnenburg, M., and Depaoli, S. (2017). A systematic review of Bayesian articles in psychology: The last 25 years. Psychological Methods, 22(2):217-239.

Wagenmakers, E.-J., Beek, T. F., Rotteveel, M., Gierholz, A., Matzke, D., Steingroever, H., Ly, A., Verhagen, J., Selker, R., Sasiadek, A., Gronau, Q. F., Love, J., and Pinto, Y. (2015). Turning the hands of time again: A purely confirmatory replication study and a Bayesian analysis. Frontiers in Psychology, 6 .

Wagenmakers, E.-J., Wetzels, R., Borsboom, D., van der Maas, H. L. J., and Kievit, R. A. (2012). An Agenda for Purely Confirmatory Research. Perspectives on Psychological Science, 7(6):632-638.

Wald, A. (1945). Sequential Tests of Statistical Hypotheses. The Annals of Mathematical Statistics, 16(2):117-186.

Wasserstein, R. L., Schirm, A. L., and Lazar, N. A. (2019). Moving to a World Beyond "p $<0.05 "$. The American Statistician, 73(sup1):1-19.

Yu, E. C., Sprenger, A. M., Thomas, R. P., and Dougherty, M. R. (2014). When decision heuristics and science collide. Psychonomic Bulletin $\&$ Review, 21(2):268-282. 